\title{
Importancia del emprendimiento social en Colombia.
}

\author{
Mónica Fernanda Chica ${ }^{1}$ \\ María Isabel Posso $\mathrm{Ch}^{2}$. \\ Julio Cesar Montoya R. ${ }^{3}$
}

\begin{abstract}
Resumen
El emprendimiento social permite la integración de actores y agentes socioeconómicos, tanto de las esferas públicas como privadas. Esta forma de emprendimiento hace posibles nuevas formas y expresiones de innovación social dando lugar a nuevos modelos de negocios. En el presente artículo se realiza una revisión teórica de los aspectos relacionados con el emprendimiento social y como ha sido su desarrollo en el contexto colombiano.

Para el desarrollo del presente trabajo que tiene como objetivo identificar la importancia del emprendimiento social en Colombia, recurrió a la búsqueda información secundaria. La recolección de información en su mayoría fue consultada en artículos y trabajos investigativos. Como resultado y partiendo de los hallazgos encontrados, el emprendimiento social es de suma importancia en un País como Colombia, puesto que a través de este se puede posibilitar el desarrollo humano y económico.
\end{abstract}

Palabras clave: Emprendimiento social, emprendedor, cambio social, proyección social, desarrollo.

\section{Introducción}

El emprendimiento social es un área de interés relativamente reciente. Podría decirse que su estudio no trasciende dos décadas, según Pastor (2011), Bill Drayton fundador de Ashoka, comienza a hablar sobre el tema como una forma de abordar problemas sociales a través de plataformas empresariales.

\footnotetext{
${ }^{1}$ Psicología, Especialista en Pedagogía para el Desarrollo del Aprendizaje Autónomo y Magister en Administración de las Organizaciones. Docente Universidad Nacional Abierta y a Distancia - UNAD monica.chica@unad.edu.co

${ }^{2}$ Psicóloga, Especialista en pedagogía del desarrollo del aprendizaje Autónomo, Magister en Administración de organizaciones. Docente. Universidad nacional Abierta y a Distancia- UNAD. maria.posso@unad.edu.co
}

${ }^{3}$ Administrador de Empresas, Especialista en Pedagogía para el Desarrollo del Aprendizaje Autónomo, Maestría en Administración de Empresas. Investigador asociado del grupo de investigación llama. Docente de la Universidad Nacional Abierta y a Distancia - UNAD. julio.montoya@unad.edu.co 
Desde su perspectiva, el emprendimiento social lo constituyen organizaciones que trabajan en programas de proyección social en donde al interior de la misma se usan conceptos administrativos y empresariales.

Es de resaltar, que siempre que se habla de este concepto se remite a las obras sociales con población vulnerable que realizan empresas como parte de la Responsabilidad social.

Sin embargo, este término va más allá, de acuerdo como Fournier (2001), se puede designar como emprendimiento social cualquier acción, iniciativa o movimiento dentro de la esfera socioeconómica, de origen privado y orientado a favor del interés general y del bien común o que beneficie a una franja significativa de la comunidad.

Para dicho fin, se requiere participar en un desarrollo sostenible y generar valor no solamente económico sino también social y humano, Por medio de la práctica del emprendimiento social, se brindan herramientas innovadoras en materia de desarrollo empresarial y humano, análisis de mercados, acompañamiento de proyectos y participación en el progreso del país.

Teniendo en cuenta la poca claridad sobre estos procesos, surge el interés del presente artículo, el cual tiene como fin realizar una revisión teórica del emprendimiento social en Colombia, para lo cual se realiza una descripción que incluye actores, la cultura, el entorno legal e institucional, determinando las esferas económicas en las cuales el emprendimiento social ocupa un espacio privilegiado en el país. Como lo son el sector público y el mundo empresarial a través de la Responsabilidad Social Empresarial ("RSE").

\section{Concepto de emprendimiento social}

A continuación se expone el concepto de emprendimiento social desde la postura de diferentes autores que han abordado el tema.

Fournier, (2011), realiza una revisión desde un punto de vista histórico, ello significa que el emprendimiento social siempre ha existido en la medida en que se puede considerar como emprendimiento social cualquier acción cometida por una agrupación social en busca de la satisfacción de una necesidad compartida por todos los miembros de la agrupación y para la cual cada miembro está dispuesto a poner en común y compartir los recursos que hacen posible la realización de la meta conjuntamente identificada.

De acuerdo con él, Banco Interamericano de Desarrollo (2006), las empresas, en forma independiente o a través de alianzas con organizaciones gubernamentales y de la sociedad civil, pueden contribuir a acelerar el proceso de mejora de la humanidad. Por su puesto, las empresas aportan al bienestar social mediante la provisión de los productos y servicios que los consumidores necesitan, la generación de empleo y el pago de impuestos para solventar los servicios públicos.

La visión de Ashoka proyecta un mundo en el que todas las personas tienen un papel como impulsores de cambios significativos para la mejora de la sociedad. En su misión de contribuir al sector, Ashoka plantea no juzgar o medir la calidad en términos 
abstractos sino apoyar el crecimiento del sector identificando y apoyando iniciativas que se basan en uno de los factores de calidad más notables: la innovación.

Hablar de emprendimiento demanda, en el caso específico de Ashoka, diferenciarlo del emprendimiento de negocios. La razón de ser de Ashoka es la profesionalización del Emprendedor Social como agente de cambio en el mundo. Para Ashoka el emprendimiento debe ser innovador, de gran impacto y con una clara estrategia de desarrollo a largo plazo; Ashoka considera que este es el modelo de emprendimiento social que está, efectiva y eficientemente, cambiando sistemas de solución a diversos problemas en el mundo. (Roa, M, s.f)

Para Roberts y Woods (2005), citados por Guzmán y Trujillo (2008) señala que “el emprendimiento social es la construcción, evaluación y persecución de oportunidades para el cambio social transformativo llevado a cabo por individuos visionarios, apasionadamente dedicados".

Ramírez (2008), indica que no existe una definición única y universal sobre emprendimiento social. Ni tampoco existe un único tipo. Sin embargo, todo emprendimiento social incluye tres elementos claves: un objetivo social, una innovación transformadora y un modelo de negocios sostenible. El emprendimiento social suele darse, y tener éxito, en los sectores de la economía donde el mercado ha fallado y la acción del Estado es inexistente o ineficaz. Un emprendimiento social, cuando resulta exitoso, puede no sólo transformar las vidas de los beneficiarios, sino también la forma de abordar un problema en general.

Según el concepto de emprendimiento social en que se basa la investigación del Banco Interamericano de Desarrollo (2006), las empresas, en forma independiente o a través de alianzas con organizaciones gubernamentales y de la sociedad civil, pueden contribuir a acelerar el proceso de mejora de la humanidad.

Por su parte, Martin y Osberg (2007) consideran, que el emprendimiento social sigue tres componentes: (1) la identicación de un equilibrio estable pero intrínsecamente injusto que causa exclusión, marginalización, o sufrimiento a un segmento de la humanidad que carece de medios nancieros o inuencia política para alcanzar un benecio transformador por su cuenta; (2) la identicación de una oportunidad en este equilibrio injusto, desarrollando una proposición de valor social y llevando a utilizar inspiración, creatividad, acción directa, coraje y fortaleza, desaando de ese modo la hegemonía del estado estable; y (3) la creación de un equilibrio estable y nuevo que libera el potencial coartado o alivia el sufrimiento del grupo objetivo, y a través de la imitación y la creación.

Las anteriores definiciones permiten tener una comprensión más amplia del emprendimiento social, entendiéndolo como un tipo especíco de emprendimiento que busca soluciones para problemas sociales a través de la construcción, evaluación y persecución de oportunidades que permitan la generación de valor social sostenible, alcanzando equilibrios nuevos y estables en relación con las condiciones sociales, a través de la acción directa llevada a cabo por organizaciones sin ánimo de lucro, empresas u organismos gubernamentales. (Guzmán y Trujillo, 2008)

Una vez clarificado el concepto, es importante comprender las características y clasificación del emprendedor social. 
De acuerdo (Roa, M, s.f), representante de Ashoka, indica que son 3 las características de los emprendedores Sociales, innovación, estrategia, e impacto. Por otra parte, de acuerdo a la revisión bibliográfica, se identifican diferentes clases de emprendedores sociales.

Otro concepto interesante es: en el que los emprendedores sociales son entendidos como "fuerzas transformadoras"; agentes con nuevas ideas para abordar problemas importantes y que son incansables en la consecución de sus ideas, gente que sencillamente no aceptará un "no" por respuesta, que no abandonará hasta que haya difundido sus ideas todo lo posible" (Bornstein, 2005, p. 96).

Sánchez (2008), afirma que los emprendedores se pueden clasificar en dos categorías en emprendedores económicos y sociales. Los primeros están totalmente orientados hacia el mercado comercial, mientras que los segundos se adhieren a conceptos ligados a la búsqueda del bien común. El mercado del emprendedor social es la población del mundo con sus diversos retos y necesidades, tomando por estandarte conceptos como: la educación básica, la igualdad de géneros, la reducción de la mortandad infantil, la lucha contra las enfermedades, la sustentabilidad del ambiente, la mejora en la calidad de vida, entre otros. Otro elemento distintivo es la carencia de afán lucrativo como fin principal de sus esfuerzos.

De acuerdo a Soto y Cárdenas (2007), citados por Mera, (2014), los emprendedores sociales tienen características muy particulares y únicas, son aquellos que ven un problema crítico en su contexto, deciden resolverlo con una visión innovadora, cuestionan el status, explora nuevas oportunidades, no acepta un no como respuesta, a la vez transforman visiones en realidades, demostrando nuevos caminos para resolver problemas y poner en marcha soluciones a gran escala.

Por otra parte, Fournier, (2014), indica que el emprendimiento social tiene formas, implicaciones y aplicaciones muy diversas y se desarrolla en las tres esferas de lo privado, lo público y lo social.

Druker, citado por Klimenko, O. (2012). destaca seis características que distinguen a un emprendedor social con éxito:

1. Disposición para corregir el punto de vista propio. Contar con esta característica implica contar con un alto nivel de flexibilidad cognitiva y personal, capacidad de aprender de los errores y humildad frente a la vida y el conocimiento.

2. Disposición para compartir los méritos. Para ser capaz de alcanzar esta característica es necesario también contar con generosidad del espíritu, nobleza del carácter, respeto por el otro, comprensión de la igualdad y del potencial humano compartido. 
3. Disposición para desligarse de las estructuras dominantes. En este aspecto es importante que la persona cuenta con capacidad de encontrar el camino propio, autonomía, tenacidad con cierto grado de terquedad, criterio propio sólido y capacidad de juicio.

4. Disposición para atravesar fronteras disciplinares. Esta disposición se basa en la presencia de una visión amplia sobre las cosas, recursividad, creatividad. 5. Disposición para trabajar tranquilamente. Aquí las personas deben contar con persistencia, paciencia, confianza en sí mismos, autoestima adecuada y un proyecto personal sólido.

6. Un fuerte impulso ético. Este impulso se basa en un compromiso con el prójimo, capacidad de comprender el dolor ajeno, empatía y altruismo como sentimientos humanos más nobles. Esta característica distingue a los emprendedores sociales como seres humanos que están más cerca de la verdadera esencia de la humanidad.

Luego de esta descripción de las características del emprendedor social, es interesante revisar cómo ha sido la evolución y el campo de acción tanto a nivel mundial como en contexto colombiano.

Siguiendo a Franco, (2014), indica que en el 2009 el Global Entrepreneurship Monitor realizó una medición acerca de las actividades que se adelantan en diferentes países del mundo. Las cifras de emprendimiento social a nivel mundial son bajas. En los países en donde GEM emprendimiento social realizó su primera medición, no se identifican un total de emprendimientos sociales superiores a un 5\%. Por regiones las que más se destacan son los Estados Unidos y el Caribe con un total igual al 5\%. América Latina tiene un promedio de $3,1 \%$,

En lo que concierne a América Latina, Colombia ocupa el segundo lugar en la realización de emprendimientos sociales (5\%), después de Argentina que con un 7,6\% presenta el porcentaje más alto de desarrollo de este tipo de emprendimientos tanto en América Latina, como a nivel mundial.

Venezuela presenta un mayor porcentaje de emprendimientos sociales nacientes, con un 3,8\% pero en el momento de pasar a la siguiente etapa este número disminuye considerablemente $(0,3 \%)$. En Brasil el total del número de emprendimientos es muy bajo $(0,4 \%)$, así como en Guatemala $(0,5 \%)$ y Ecuador $(0,7 \%)$.

\section{Emprendimiento social en Colombia.}

Las cifras que presenta GEM emprendimiento social (2011) muestran datos interesantes en cuanto a la consolidación de emprendimientos sociales en Colombia. Junto con Argentina son los países con mayor porcentaje de emprendimientos en etapa inicial y/o emprendimientos ya establecidos. (Franco, 2014, p.p 11).

En Colombia la actividad de emprendimiento social ha venido desarrollándose de manera importante desde inicios del presente siglo.

Entidades gubernamentales como ANSPE a través de su red de proyectos sociales y su actividad Hilando Proyectos, apoya diferentes agrupaciones que realizan 
emprendimientos sociales entre las que se cuentan Ahmsa, la Fundación Alpina, la Fundación Social, entre otras.

De igual forma, Ashoka, ha venido desarrollando su modelo y cuenta con varios proyectos que tienen que ver con educación, medio ambiente, participación ciudadana. Estos proyectos son llevados a cabo por asociados que tiene fundaciones como Abcdespañol, creada por Javier González, que a partir elementos lúdicos ha enseñado a leer y a escribir a un millón de niños. Luis Camargo, de la Fundación OpePA que busca conectar a los jóvenes de ambientes urbanos y rurales con la tierra, a la vez que trabaja en el impulso de agentes de cambio; Juan David Aristizabal con su Fundación Ideas por un País Mejor, entre otros. (Franco, 2014, p.p 11).

De acuerdo con el Departamento Nacional de Planeación, Política de Innovación Social, (2014), Colombia no es ajena a esta compleja problemática socio-económica y la innovación social representa una opción pertinente para superar los enfoques tradicionales que tienen los programas y proyectos del Estado.

Es por ello que desde 2012 se ha venido trabajando, a partir de un proceso de construcción colectiva e interinstitucional del Nodo Nacional de Innovación Social, en la formulación de la política pública de innovación social en la cual se definió como IS “el proceso a través del cual se crea valor para la sociedad mediante prácticas, modelos de gestión, productos o servicios novedosos que satisfacen una necesidad, aprovechan una oportunidad y resuelven un problema de forma más eficiente y eficaz que las soluciones existentes, produciendo un cambio favorable en el sistema en el cual opera. La IS se caracteriza por tener potencial de escalabilidad, replicabilidad, ser sostenible, promover mayores niveles de empoderamiento de la comunidad y generar alianzas entre diferentes actores de la sociedad".

Siguiendo a Gutiérrez, (2007), el empresariado en Colombia lidera y participa en múltiples iniciativas con impacto en la sociedad. Sus contribuciones tienen una trayectoria histórica destacada. En la década de 1960 surgieron fundaciones de origen empresarial que hoy se mantienen y sobresalen por la cantidad y calidad de sus intervenciones.

La inversión social de las empresas, aunque no tiene relación directa con su negocio, genera beneficios como imagen, compromiso y sentido de pertenencia de sus empleados. En Colombia hay por lo menos tres modelos diferentes de inversión de las empresas en la sociedad: i) unas han decidido crear sus propios programas al interior de la empresa; ii) otras han decidido crear una organización aparte (e.g. una fundación, una corporación o una asociación) o apoyar una organización sin fines de lucro ya existente; y iii) algunas más participan en programas sociales liderados por otros (i.e. iniciativas de un gremio, de una organización sin fines de lucro ya existente, de una red específica o del gobierno de turno).

Las fundaciones empresariales y las fundaciones con empresas tienen una estrecha relación con una empresa particular y su rango de acción es amplio. (Gutierrez, 2007, p.p. 25).

Continuando con el autor, indica que en Colombia existe una gran diversidad de iniciativas que responden a distintas necesidades, están estructuradas de diferentes maneras y tienen impacto en trabajadores, proveedores, clientes, competidores, comunidad local y sectores específicos de la sociedad. Para poder comprender mejor el amplio espectro de estas iniciativas, se pueden agrupar en tres tipos:

1. Iniciativas filantrópicas alejadas del negocio. 
2. Emprendimientos sociales integrados al negocio.

3. Contribuciones a la construcción de instituciones y políticas públicas.

\section{Conclusiones}

En este artículo se ha resaltado el concepto del emprendimiento social desde diferentes autores, asimismo como las características del emprendedor social, se identificó además, el objeto principal de este tipo de emprendimiento el cual consiste en la búsqueda y puesta en marcha de soluciones a problemas sociales, para esto se requiere de la acción directa de los emprendedores sociales.

Este emprendimiento no está circunscrito únicamente al sector de las organizaciones sin ánimo de lucro, sino que debe ser llevado a cabo por otro tipo de organizaciones tales como los organismos gubernamentales o por las empresas del sector privado.

El éxito del emprendimiento social está en el impacto que éste tiene sobre el desarrollo económico, y es especialmente oportuno en países en vías de desarrollo, en los que la construcción social colectiva complementa la acción realizada por el Estado para hacer frente a los problemas de la sociedad.

En Colombia hay un avance significativo e interés de la empresa privada por ejercer este tipo de actividad a través de la conformación de fundaciones que propenden por el desarrollo humano, enmarcado en las políticas públicas.

\section{Bibliografía}

Arbeláez, L. (2011). Emprendimiento social: factores críticos de éxito y fracaso para el mejoramiento del programa de generación de ingresos de la presidencia de la república de Colombia, dirigido a población en situación de desplazamiento por la violencia en caldas. Universidad nacional de Colombia. Sede Manizales.

Banco interamericano de desarrollo. (2006). Gestión efectiva de emprendimientos sociales. Lecciones extraídas de empresas y organizaciones de la sociedad civil en Iberoamérica. Un proyecto de investigación colectiva de la Social Enterprise Knowledge Network. Editorial Planeta.

Bornstein, D. (2005). Como cambiar el mundo: el poder de los emprendedores sociales. Barcelona: Limperfraf. 
Departamento Nacional de Planeación, Política de Innovación Social, (2014). Innovación y emprendimiento social: alternativa de alto impacto para la construcción de un nuevo modelo de desarrollo para el país. Recuperado de: www.fondoemprender.com/.../INNOVACION_Y_EMPRENDIMIENTO_SOCIAL.PD

Fournier, A. (2001). Emprendimiento social. Documentos de Administración. Universidad Central. Recuperado de: https://www.ucentral.edu.co/images/documentos/editorial/2015_emprendimiento_social_00 $\underline{1 . p d f}$

Franco, P. (2014). El emprendimiento social al interior del centro de emprendimiento UR emprende de la universidad del rosario. Trabajo de grado. Maestría en estudios sociales. Universidad del Rosario

Gutiérrez, R. (2007). Emprendimientos sociales En sectores Estratégicos de desarrollo En Colombia. Iniciativa en emprendimientos Sociales, IESO, Facultad de administración de la universidad de los andes.

Guzmán, A; y Trujillo, M. (2008). Emprendimiento social - Revisión de literatura. Estudios gerenciales Vol. 24 No. 109.

Klimenko, O. (2012). Emprendedores sociales: un camino desde la postura personal hacia la resiliencia comunitaria. Revista Psicoespacios, Vol 6. N. 8, pp. 275-286, Disponible en http://www.iue.edu.co/revistasiue/index.php/Psicoespacios/article/view/113

Martin, R. y Osberg, S. (2007). Social Entrepreneurship: The case for denition. Stanford social innovation review, 5(1), 28-39.

Mera, C. (2014). Prospectiva del Emprendimiento Social y Solidario «Retos y Desafios para la Construcción Social de Territorios de Futuro». Universidad Nacional Abierta y a Distancia. Bogotá, D.C. Colombia.

Pasto, G. (2011). Emprendedores Sociales: Bill Drayton, Fundador Ashoka. Recuperado de http://www.emprendedorsocial.org/emprendedores-sociales-bill-drayton-fundadorashokal

Roberts, D. y Woods, C. (2005). Changing the world on a shoestring: The concept of social entrepreneurship. University of Auckland Business Review, 7 (1), 45-51.

Roa, M. (s.f). Definición y alcances del emprendimiento social. Representante de Ashoka en Colombia. Ashoka Emprendedores Sociales recuperado de: www.espanol.ashoka.org 
Ramírez, A. (2008). ¿Qué es el emprendimiento social? Concurso Emprendedor social del año América Central Recuperado de:

http://www.schwabfoundseoy.org/es/competitions/competition/75

Sánchez, A. (2009). La Formación de Emprendedores Sociales: Caso Tecnológico de Monterrey 